\title{
C'EST grave docteur ? À propos du caractère "schizophrénique » de la notion de « dignité humaine »
}

\section{Is it serious doctor? Concerning the "schizophrenic" character of the concept of "human dignity"}

\author{
É grave doutor? A propósito da característica \\ "esquizofrênica" da noção de "dignidade humana"
}

Fabien Bottini*

\section{Résumé}

Mère de toutes les libertés pour les uns, prétexte aux mesures les plus liberticides pour les autres, la dignité humaine serait tantôt un droit opposable à autrui (État ou particulier) et tantôt un droit opposé à soi-même, car tantôt un droit subjectif bénéficiant individuellement à chacun en tant qu'être humain et tantôt une obligation objective pesant collectivement sur chaque membre de l'espèce humaine. Le concept semble ainsi souffrir de schizophrénie, affection grave s'il en est tant elle peut être à l'origine de bien des malheurs. Mais cette dualité de fonctions est-elle aussi intrinsèquement liée à la notion qu'il n'y parait ? Tout dépend de la conception du droit que l'on retient. Car autant les approches jusnaturaliste classique et positiviste juridique permettent d'accréditer cette idée, autant les conceptions jusnaturaliste moderne et positiviste sociologique conduisent à la rejeter. Leur confrontation est ainsi riche d'enseignements. Non seulement elle renseigne sur l'évolution du regard que l'homme a porté sur lui à travers l'histoire, mais elle révèle l'une de ces luttes dialectiques qui animent le droit positif contemporain. Tout se passe en effet comme si les dépositaires du pouvoir empruntaient via le positivisme juridique à la conception jusnaturaliste classique l'idée que des "instances supérieures " peuvent limiter le pouvoir d'autodétermination des individus sur eux-mêmes. A l'inverse, l'opinion publique semble au contraire retenir de la conception jusnaturaliste moderne l'idée qu'il revient à chacun de déterminer en raison ce que commande la notion, pour en faire collectivement une garantie efficace, non plus seulement de sa seule

Docteur en droit. Maître de conférences à l'Université du Havre. Le Havre-Normandie-France. Email: fabienbottini@yahoo.fr 
liberté autonomie, mais de façon plus générale de ses droits et libertés les plus essentiels.

Mots-clés: Dignite humaine. Positivisme juridique. Positivisme sociologique. Jusnaturalisme.

\section{Abstract}

Mother of all freedoms for some, pretext for the most liberticide measures for others, human dignity is sometimes a right against another (state or individual), and sometimes a right opposed to itself. This because it is sometimes a subjective right benefiting to each individual as a human being and sometimes an objective obligation collectively weighing on every member of the human species. Thus the concept seems to suffer from schizophrenia. Is this dual function really intrinsically tied to the notion as it seems? Everything depends of the conception of law that we accept. Everything happens indeed as if the rulers through legal positivism borrowed to the classic natural law the idea that "higher authorities" can limit the freedom of individuals. In contrast, public opinion seems rather borrowing to the modern natural law the idea that it is for each one to determine rationally what the notion commands, to use it as a collective guarantee of the most essential rights and freedoms.

Keywords: Human dignity. Schizophrenia. Legal positivism. Natural law.

\section{Resumo}

Mãe de todas as liberdades para alguns, pretexto para medidas destruidoras da liberdade para outros, a dignidade humana seria tanto um direito oponível a terceiros (estado ou particular), quanto um direito oponível a si mesmo, ou, por vezes, um direito subjetivo beneficiando individualmente enquanto ser humano ou ainda uma obrigação objetiva pesando coletivamente sobre cada membro da espécie humana. O conceito parece, assim, sofrer de esquizofrenia, que pode estar na origem de muitos infortúnios. Mas esta dualidade de funções é também intrinsecamente ligada à noção não Ihes são próximas? Tudo depende da noção de direito que é escolhida. Tanto as abordagens jusnaturalistas clássicas e do positivismo jurídico permitem confirmar esta idéia, quanto as concepções jusnaturalistas modernas e do positivismo sociológico conduzem a rejeitá-la. Sua confrontação é rica em ensinamentos. Ela fornece não apenas informações sobre a evolução do olhar que o homem lança sobre si mesmo ao longo da história, como também revela uma das lutas dialética que animam o direito positivo contemporâneo. Tudo se passa, com efeito, como si os depositários do poder emprestassem à concepção jusnaturalista clássica 
via positivismo jurídico a idéia de que "instâncias superiores" podem limitar o poder de autodeterminação dos indivíduos sobre eles mesmos. Ao inverso, a opinião publica parece, ao contrário, reter da concepção jusnaturalista moderna a idéia de que caberia a cada um determinar, em razão do que comanda esta noção, para assegurar uma garantia eficaz, não mais apenas de sua própria liberdade autônoma, mas de modo mais genérico de seus direitos e liberdades mais essenciais.

Palavras-chave: Dignidade humana. Esquizofrenia. Positivismo jurídico. Jusnaturalismo.

\section{Introduction}

Si «la force et le droit (...) règlent toutes choses dans le monde», la dignité humaine semble bien être l'un des enjeux de leur affrontement (JOUBERT, 1850, p. 355).

Aussi loin qu'elle puisse remonter, l'histoire du droit montre d'ailleurs que la force n'a pas hésité à instrumentaliser le droit pour nier la dignité de certains hommes. Que l'on songe à l'esclavage sous l'Antiquité (CRUVEILHIER, 1938, p. 245) ou à la Shoah née des atrocités nazies sous la seconde guerre mondiale sans oublier l'asservissement des peuples colonisés au XVI ${ }^{\mathrm{e}}$ siècle (CARRIERE, 1999), on se rend compte que de nombreuses exactions contre les personnes ont été juridiquement fondées.

Sans doute l'histoire récente traduit-elle une certaine involution dans cette lutte, le droit tendant depuis la seconde moitié du $X^{\mathrm{e}}$ siècle à instrumentaliser la force pour assurer la sauvegarde de la dignité humaine, tant au niveau international qu'interne ${ }^{1}$. Au point que certains auteurs n'hésitent pas à faire de la dignité humaine le principe juridique suprême : “Grundprinzip » (VAUCHEZ-HENNETTE, 2009, p. 72); « principe supraconstitutionnel » (RIALS, 1986, p. 57) ; " socle des droits fondamentaux » (RENUCCI, 1999, p. 2); "droit éminent »

V. par ex. L. fondamentale allemande du 23.5.1949, Pr. art. 1er (ALLEMAGNE, 1949) 
(JORION, 1999, p. 225), « principe matriciel » (MATHIEU, 1995, p. 211) ... les qualificatifs ne manquent pas pour désigner son importance dans l'ordonnancement normatif. Mais son influence reste relative.

Tout d'abord, parce qu'il n'y a guère qu'une cinquantaine d'États sur les 192 membres que compte l'ONU à satisfaire les critères de la démocratie qui seuls semblent à même d'assurer son respect. Ensuite, parce que cette cinquantaine de pays n'est pas à l'abri de phases de "régressions », comme l'attestent le recours au Waterboarding - ces simulacres de noyade - par la CIA américaine dans le cadre de la « guerre contre le terrorisme »(LE MONDE, 2009). Certaines puissances, à l'image de la France, ont d'ailleurs tardé à constitutionnaliser le principe.

Aussi surprenant que cela puisse paraître, il aura en effet fallu 156 années à la patrie des droits de l'homme pour hisser la dignité humaine au sommet de sa hiérarchie des normes: consacrée par le pouvoir administratif en 1848, le terme ne s'est vu reconnaître rang de PGD par le juge ordinaire que dans la première moitié du $X X^{e}$ siècle (FRANCE, 1942, p. 63), le législateur ne le consacrant que dans les années 1980 avant que le juge constitutionnel ne se décide enfin à l'intégrer au « bloc de constitutionnalité » en 1994, en lui reconnaissant rang de « principe à valeur constitutionnelle ».

Cette constitutionnalisation apparait d'autant plus tardive que la notion était bien connue de l'histoire des idées politiques. Outre que la pensée judéo-chrétienne s'est efforcée au point de vue religieux de prêcher son respect, la philosophie n'a eu de cesse de militer en faveur de sa défense, tant sous l'Antiquité ${ }^{2}$, les Temps Modernes qu'à l'époque des Lumières. C'est ainsi qu'au XVIII siècle, Voltaire se disait favorable à ce que « tombe avec le tyran tout ce qui peut (...) servir à dégrader la dignité de l'homme » tandis que Montesquieu (1877, p. 365) dénonçait les «États où les lois (...) ne laissent aucune dignité (...) aux sujets » ${ }^{3}$.

\footnotetext{
2 Avec le stoïcisme (apparu au IVe siècle A. E.), d'après lequel " l'homme » a " une dignité » qui tient à "l'insigne honneur » qui lui « a été fait d'être composé d'une Âme faisant partie de l'essence divine » (LABORDERE, 1903, p. 87).

3 De l'Esprit des lois, C. Delagrave 1887. 14.
} 
La récupération de la notion par le droit permet néanmoins de lui donner une certaine effectivité. C'est particulièrement vrai en France où sa constitutionnalisation se combine désormais avec un contrôle de constitutionnalité permettant à chacun d'assurer son respect.

II n'est pas étonnant dans ces conditions que la dignité de la personne humaine soit une notion en pleine expansion. Certains débats sont d'ailleurs propices à son invocation, tels que ceux désormais récurrents qui accompagnent la révision (théoriquement) quinquennale des lois de bioéthique. Entre autres, le législateur de 2010 devra ainsi s'interroger sur l'opportunité d'autoriser plus avant la recherche sur l'embryon et la recherche biomédicale ou encore de légaliser le recours aux « mères-porteuses » - aux « surrogate mothers » comme disent les anglo-saxons - actuellement interdit. Autant de questions qui mettent en jeu la dignité humaine.

Cette utilisation à tout va brouille toutefois quelque peu la compréhension de l'expression. La controverse sur la légalisation éventuelle du recours aux mères de substitution est particulièrement représentative de cet état de fait. La gestation pour autrui renvoie à une technique destinée à satisfaire l'aspiration - compréhensive - des couples incapables de procréer, du fait d'un problème utérin de la mère d'intention, à avoir un enfant qui peut être biologiquement le leur. Mais à la question : " faut-il ou non autoriser le recours aux mères porteuses ? " la dignité de la personne humaine est également invoquée par les partisans du « non » et ceux du « oui ».

Ces ambigüités autour de sa signification ne font que refléter celles qui entourent sa fonction. Mère de toutes les libertés pour les uns (MATHIEU, 1996, p. 282), prétexte aux mesures les plus liberticides pour les autres' la dignité serait tantôt un droit opposable à autrui (État ou particulier) et tantôt un droit opposé à soi-même (LEBRETON, 2000, p. 60) car tantôt un droit subjectif ${ }^{4}$ bénéficiant individuellement à

C'est-à-dire « une prérogative ou un pouvoir qui appartient à une personne d'obtenir un effet juridique en vertu de la règle de droit » (ROUBIER, 2005, p. 5). 
chacun en tant qu'être humain et tantôt une obligation objective pesant collectivement sur chaque membre de l'espèce humaine (LEBRETON, 2000, p. 58; EDELMAN, 1997, p. 187; MOUTOUH, 1999. p. 166; JORION, 1999, p. 209; VAUCHEZ-HENNETTE, 2009, p. 71; FEDELMAN, 2009, p. 88).

Cette ambivalente apparaît d'autant plus marquée que la dimension universelle de la notion semble cacher des " tendances catégorielles ». En tant que droit, cette dualité découle de sa vocation à bénéficier à tous malgré sa propension à protéger plus spécifiquement certaines parties de la population, notamment les personnes rendues vulnérables par leurs caractéristiques physiques - les enfants ou les malades (les nains $?^{5}$ ) - ou leur situation sociale - les salariés, les personnes défavorisées ou les étrangers (MOUTOUH, 1999, p. 174 s). En tant que devoir, cette ambigüité découlerait de sa prétention à s'imposer à toutes personnes en tant que nouveau " standard des bonnes mœurs » (VAUCHEZ-HENNETTE, 2009, p. 60) tout en pesant plus spécifiquement sur certaines en raison de l'obligation d'exemplarité que leur impose leur situation dans la hiérarchie sociale ${ }^{6}$.

Le concept semble ainsi souffrir de schizophrénie ${ }^{7}$, affection grave s'il en est tant elle peut être à l'origine de bien des malheurs. Mais cette dualité de fonctions, faisant de la dignité humaine une source tantôt de droits envers autrui et tantôt de devoirs envers soi-même, est-elle aussi intrinsèquement liée à la notion qu'il n'y parait ?

Tout semble en fait dépendre de la conception du droit à laquelle on se rallie. Car cette dualité n'est pas sans connotation axiologique implicite, dès lors que la dignité peut être envisagée comme un concept jusnaturaliste ou positiviste. Dans le premier cas, la notion peut revêtir

La question se pose en raison de la célèbre affaire du lancé de nain rappelée infra II.A.

$6 \quad$ C'est ainsi que le « dérapage » verbal du ministre de l'intérieur selon lequel « quand il y a en un [sous-entendu individu d'origine maghrébine], ça va. C'est quand il y en a beaucoup qu'il y a des problèmes » peut être considéré comme attentatoire à la dignité humaine (LEMONDE 2009)

7 Comme l'ont relevé de nombreux auteurs : CAYLA (2003); ROMAN et al (2004, p. 95); VEIL et al. (2008, p. 92) 
une acception jusnaturaliste classique, trouvant ses fondements dans les enseignements de la religion judéo-chrétienne, ou jusnaturaliste moderne, conforme aux enseignements des philosophes des Temps Modernes. Dans le second, elle peut être appréhendée comme un principe positiviste juridique ne revêtant qu'une dimension formelle ou un principe positiviste sociologique doublé d'une acception matérielle.

De prime abord, ces conceptions semblent dans un rapport d'antithèse radicale. Car, si les conceptions jusnaturalistes ont en commun de partir d'une représentation abstraite de l'être humain et d'en déduire une construction a priori du concept de dignité dont les implications sont immuables, les conceptions positivistes ont pour originalité de partir d'une réflexion concrète de l'homme sur lui-même qui les amènent à promouvoir une construction a posteriori de la notion dont les implications, loin d'être figées, sont évolutives.

Mais à l'analyse les choses ne sont pas aussi simples. Non seulement en effet les conceptions jusnaturalistes divergent entre elles quant aux conséquences qu'il convient d'attacher au principe, mais il en est de même pour les conceptions positivistes. Ces divergences viennent de ce qu'il se trouve dans chaque grande école de pensée (jusnaturaliste et positiviste) une conception pour imposer "d'en haut » les conséquences attachées au concept de dignité (celles-ci étant définies par Dieu dans la conception jusnaturaliste classique ${ }^{8}$ et par les autorités normatives dans la conception positiviste juridique) tandis qu'une autre les définit « d'en bas » (leur portée étant alors déterminée par chacun individuellement dans la perspective jusnaturaliste moderne et par tous collectivement dans la perspective positiviste sociologique). De sorte qu'il semble possible de croiser chaque conception jusnaturaliste avec une conception positiviste. Or, ce rapprochement met en lumière des conséquences différentes quant à la fonction du concept.

8 Tantôt directement, en révélant à certains hommes le contenu de la loi naturelle, tantôt indirectement, en investissant d'autres des qualités nécessaires à sa découverte, de sorte que ces derniers doivent en principe être obéis parce qu'ils sont dotés " d'une certaine compétence pour orienter les membres du corps social vers cet élément essentiel à toute vie commune que constitue le droit » (SERIAUX, 1993, p. 61). 
Autant en effet les approches jusnaturaliste classique et positiviste juridique permettent d'accréditer l'idée d'une dualité de fonctions du principe de dignité (I), autant les conceptions jusnaturaliste moderne et positiviste sociologique conduisent à son rejet, la fonction intrinsèque du principe étant alors de fonder les droits ou libertés de chacun envers autrui (II).

\section{Les conceptions Jusnaturaliste classique et Positiviste Juridique ou la confirmation d'une dualité de fonctions du Principe de Dignité}

Comme on l'a vu, les conceptions jusnaturaliste classique et positiviste juridique ont en commun de défendre une conception de la dignité qui soit imposée "d'en haut " et dont découle une dualité de fonctions, la notion étant à la fois source de droits envers autrui et de devoirs pour soi-même.

Tandis que la conception jusnaturaliste classique fonde ce caractère dual sur le commandement divin fait aux hommes de respecter la vie humaine $^{9}(A)$, la conception positiviste juridique y conduit en obligeant chacun à respecter la volonté des autorités normatives régulièrement transcrite en droit positif, indépendamment de son contenu (B).

1.1 La conception jusnaturaliste classique ou la dualité de fonctions fondée sur le respect de la vie

La conception jusnaturaliste classique fait de la dignité une source de droits à l'égard des tiers et de devoirs pour soi-même, dans la mesure où elle érige la vie en valeur suprême et où cette sacralisation a pour effet de dénier aux individus un total pouvoir tant sur les autres que sur eux-mêmes.

9 «Tu ne tueras point » trouve-t-on gravé sur les Tables de la loi (LA SAINTE BIBLE, Exode 20,13) 
Cette conception doit son nom à son rattachement à l'école classique du droit naturel. Selon ses membres, il existe des droits qui s'imposent au droit positif, entendu comme le droit formulé par les autorités humaines habilitées à le poser ${ }^{10}$. Les deux explications avancées à l'appui de cette prééminence correspondent au double héritage de la pensée occidentale, gréco-romain et judéo-chrétien. Elles s'accordent pour considérer l'individu comme la partie d'un tout et sur l'idée d'un droit naturel objectif, pouvant être découvert via la raison pratique par l'observation de l'Univers. Mais elles s'opposent quant à la justification de la primauté de ce droit naturel.

Tandis que la pensée gréco-romaine la déduit de son origine mystique, du fait qu'il est le reflet des lois de la nature ${ }^{11}$, I'héritage judéochrétien y voit la conséquence de son origine métaphysique, les droits naturels étant à ses yeux le produit de la volonté divine (AQUIN, 1862, p. 213).

De là des divergences quant à leurs implications. La pensée grécoromaine n'en déduit pas l'existence d'une dignité propre à la personne humaine pour la bonne et simple raison que l'individu n'existe d'après elle qu'à travers la communauté dont il est issu, cette dernière ayant tout pouvoir sur lui' ${ }^{12}$. À l'inverse, la religion judéo-chrétienne fait d'une telle dignité la condition même de l'être humain dès lors que Dieu a fait I'homme à son image (LA SAINTE BIBLE, Gen. 1, 26 et 27). Aucun doute n'est permis sur ce point dans le catholicisme, dans la mesure où le Saint-Siège a eu l'occasion d'affirmer expressément que « c'est l'image de Dieu dans l'homme qui fonde (...) la dignité de la personne humaine » (VATICAN, 1986, $\mathrm{n}^{\circ} 27$ ).

L'Église en tire une conséquence importante, puisqu'elle en conclut que nul n'a le droit de porter atteinte à la vie humaine. Cette

\footnotetext{
10 «On appelle positif ce qui procède de la volonté humaine » (AQUIN, 1862, p. 211).

11 «Ce qui est d'origine naturelle est ce qui en tous lieux a le même effet et ne dépend pas de nos diverses opinions » (ARISTOTE, 1897, v. 7).

12 Le stoïcisme faisant figure d'exception.
} 
idée se trouve exprimée dès les premières pages de la Bible, d'après laquelle quiconque fait couler le sang de l'homme encourt en principe la colère divine. Par exception, les textes saints enseignent que Dieu se reconnait la faculté d'ôter la vie de ceux qui le défient, tantôt directement - comme cela ressort du récit du Déluge, par lequel se trouvent emportés tous les hommes souillés par le péché à l'exception de Noé et de ses descendants ${ }^{13}$ - ; et tantôt par procuration ${ }^{14}$. Mais si celui « qui verse le sang de l'homme, par l'homme aura son sang versé », c'est toujours parce qu' "à l'image de Dieu l'homme a été fait " (LA SAINTE BIBLE, Gen. 9,6). Ainsi, comme le résume la papauté, le principe est et demeure que " tout ce qui s'oppose à la vie elle-même " est " infâme[] " ${ }^{15}$ comme attentatoire à la dignité humaine et qu'« il n'est permis à personne de " la "violer impunément » sans en être comptable " envers Dieu » (VATICAN, 1891).

On comprend dès lors que cette variante du jusnaturalisme classique assigne une dualité de fonction au concept de dignité humaine. Celle-ci est source de droits subjectifs, dès lors qu'elle garantit le droit à la vie de chacun. Mais elle est également source de devoirs objectifs, puisqu'elle justifie de défendre aux individus de porter atteinte à leur propre dignité. L'interdiction sous peine de sanctions pénales de l'euthanasie active en droit français peut se réclamer de cette doctrine. Car la mesure reflète cette conviction chère à l'Église selon laquelle « l'homme n'est pas le propriétaire (...), mais seulement l'usufruitier » (MOUTOUH, 1999, p. 191) de son corps.

13 Gen. 2, 21-23 (LA SAINTE BIBLE) : « alors périt toute chair qui se meut sur la terre : (...) depuis l'homme jusqu'aux (...) oiseaux du ciel ». Dans le même sens, on peut citer la destruction de Sodome et Gomorrhe, dont les fautes des habitants envers le Tout-Puissant nous dit la Bible étaient « bien grave[s] » (LA SAINTE BIBLE, Gen. 18, $20 ; 19,13$ ).

14 C'est dire que l'Église catholique admet la peine de mort, dès lors que celle-ci traduit la volonté divine. Historiquement, la doctrine sacerdotaliste, symbolisée par la thèse des deux glaives de Bernard de Clairvaux, a justifié cette solution par le fait que la justice est rendue par le pouvoir temporel sur délégation du pouvoir spirituel (LECA, 1997, p. 119).

15 Concile Vatican II, Gaudium et spes, 7.12.1965, n $31 \S 3$. 
La conception jusnaturaliste classique accrédite ainsi l'idée d'une double fonction du principe de dignité. C'est ce qui la rapproche de la conception positiviste juridique, même si cette dernière aboutit au même résultat pour de toutes autres raisons.

\subsection{La conception Positiviste Juridique ou la dualité de fonctions fondée sur l'autorité du pouvoir normatif}

La conception positiviste juridique conduit à faire du principe de dignité une source de droits inter personnels autant que de devoirs intra personnels, dans la mesure où elle permet aux autorités normatives de déterminer elles-mêmes ce qu'il commande et où ces dernières n'ont pas hésité à l'invoquer pour limiter le pouvoir de chacun sur lui-même.

Le positivisme juridique nie en effet l'existence de droits naturels. Le seul droit qu'il connait est le droit positif. C'est ce qui le distingue du jusnaturalisme et ce qui explique qu'à ses yeux la dignité de la personne humaine ne puisse être que ce que l'ordonnancement juridique dit qu'elle est. Car seule compte au regard du positivisme juridique la régularité des conditions d'adoption des règles de droit positif : dès lors qu'elles sont élaborées par les autorités habilitées conformément aux règles de forme et de procédure prévues à cet effet, elles doivent être considérées comme juridiquement obligatoires. En conséquence, la science du droit doit se contenter de décrire objectivement l'ordonnancement juridique, en s'abstenant de formuler tout jugement de valeur sur son contenu.

Cette approche offre l'avantage de fonder un certain nombre de droits et libertés que chacun peut opposer à autrui sur la base d'une conception « laïcisée » du principe de dignité. Elle donne en d'autres termes aux autorités normatives le moyen de surmonter les difficultés qui peuvent survenir dans une société multiconfessionnelle du ralliement pur et simple à la conception judéo-chrétienne de la dignité humaine, en affirmant l'existence de droits sans avoir à se justifier sur leur fondement. Sans doute ces droits cessent-ils d'être subjectifs au sens jusnaturaliste classique du terme, dès lors qu'ils procèdent, non plus de l'ordre naturel 
des choses, mais de la légalité, c'est-à-dire du droit objectif ${ }^{16}$. Mais ils restent subjectifs, en ce sens qu'ils traduisent une capacité préalablement concédée à chacun par ce dernier (GRZEGORCZYK et al, 1993, p. 37).

Malgré sa rigueur, cette conception des choses n'est toutefois pas sans danger. Car blanc seing est donné à travers elle aux autorités d'utiliser le droit objectif pour limiter le pouvoir de chacun sur lui-même, au nom de la conception qu'elles se font du principe de dignité et de ses implications. Le positivisme juridique présente ainsi le défaut d'exposer les gouvernés à l'arbitraire du pouvoir, dès lors que sa maîtrise de l'ordre juridique lui permet d'imposer ses vues, au besoin en recourant à la force publique. Ce danger est d'autant plus réel que la « logique représentative ${ }^{17}$ qui sous-tend le régime représentatif conduit les autorités normatives à se comporter comme si elles étaient le véritable souverain. Un exemple relativement récent tiré de la jurisprudence du Conseil d'État montre d'ailleurs que cette dérive n'est pas qu'une hypothèse d'école.

Il tient à la décision de la haute juridiction d'ériger « le respect de la dignité de la personne humaine » en nouvelle composante de l'ordre public. Car, dans cette affaire, les conseillers d'État n'ont pas simplement recouru à la notion pour interdire un spectacle de lancé de nain. Ils l'ont fait alors même que le nain " se prêtait librement à cette exhibition, contre rémunération ${ }^{18}$. C'est pourquoi la décision a été critiquée : parce qu'elle revenait à instaurer un « ordre moral », le juge se comportant en Dieu démiurge pour limiter autoritairement le pouvoir de certaines personnes sur elles-mêmes au nom de sa propre conception de la dignité humaine (Lebreton, 2000, p. 62) ${ }^{19}$.

16 Entendu comme " une règle de conduite qui s'impose aux hommes vivant en société et dont le respect est assuré par l'autorité publique » (ROUBIER, 2005, p. 5). Le positivisme juridique reprend sur ce point les conclusions des auteurs jusnaturalistes modernes qui avaient considéré avant lui que « le droit subjectif est soumis au droit objectif dont il constitue la matière » (SERIAUX, 1993, p. 78).

17 Sur cette notion, v. Bottini (2008, p. 23).

18 Cf. CE 27.10.1995, préc.

19 Dreyer (2008, p. 2730) : dénonçant une « conception (...) de la dignité » qui « repose sur la vision d'un homme infantilisé » et traduit « une dérive fondamentaliste », un « néocolonialisme culturel permet[ant] au clerc d'imposer sa lumière ». 
Quoique pour des raisons différentes, les conceptions positiviste juridique et jusnaturaliste classique assignent ainsi une double fonction au principe de dignité, ce dernier étant selon elles source tantôt de droits envers autrui et tantôt de devoirs envers soi-même. C'est ce qui les distingue des conceptions jusnaturaliste moderne et positiviste sociologique, ces dernières faisant intrinsèquement du principe de dignité une source de droits envers autrui.

\section{Les conceptions jusnaturaliste moderne et Positiviste Sociologique ou le rejet d'une dualité de fonctions du principe de dignité}

Le jusnaturalisme moderne et le positivisme sociologique promeuvent une conception du principe de dignité définie "d'en bas », dont l'originalité est de rejeter toute idée d'une dualité de fonctions du principe de dignité, ce dernier étant avant tout une source de droits envers autrui.

Tandis que la conception jusnaturaliste moderne justifie cette condamnation par la nécessité de respecter la liberté-autonomie de chacun (A), la conception positiviste sociologique la fonde sur la nécessité de respecter l'attachement de la conscience collective aux droits et libertés essentiels de l'individu (B).

2.1 La conception jusnaturaliste moderne ou le rejet d'une dualité de fonctions fondé sur la liberté-autonomie

La conception jusnaturaliste moderne s'oppose à tout dédoublement fonctionnel de la dignité humaine, dans la mesure où elle érige la liberté-autonomie ${ }^{20}$ en valeur suprême ${ }^{21}$ et où cette sacralisation a pour conséquence de reconnaître aux individus un total pouvoir sur

\footnotetext{
20 C'est-à-dire à « la sphère d'autonomie individuelle du gouverné, résultant de l'interdiction faite au gouvernant de franchir certaines limites » (LEBRETON, 2009, p. 12).

21 En ce sens, v. Cayla (1998, p. 154).
} 
eux-mêmes, un pouvoir d'autodétermination absolu impropre à fonder l'existence de devoirs dans la relation avec soi.

L'avènement de cette conception alternative est indissociable de celui de la conception « moderne » de la liberté, théorisée par Benjamin Constant à l'occasion de son discours devant l'Athénée royal de Paris en $1819^{22}$. L'apparition de cette dernière trouve elle-même son origine dans les réflexions du XVI ${ }^{\mathrm{e}}$ siècle. Annoncée par les penseurs italiens et allemands, elle est l'œuvre des philosophes anglais.

Les premiers ont préfiguré cette évolution en émancipant l'homme des contraintes extérieures qui pesaient jusque là sur lui. C'est au Florentin Nicolas Machiavel (1469-1527) qu'il est revenu d'ouvrir la voie à ce changement. Cherchant un fondement à l'absolutisme monarchique, il suggère en 1513 aux rois d'asseoir leur pouvoir « par force ou par ruse » (MACHIAVEL, 1884, p. 80), en profitant notamment de l'antagonisme du peuple et de la noblesse (MACHIAVEL, 1884, p. 76). Ce faisant, il prépare l'autonomisation des dépositaires de la Couronne, leur liberté, par rapport aux institutions auxquelles ils étaient jusqu'alors soumis : l'Empire et l'Église romaine à l'extérieur et la féodalité à l'intérieur (CARRE DE MALBERG, 1920, p. 73). Si cette libération ne devait dans son esprit profiter qu'au Trône, la pensée allemande va étendre son bénéfice à l'ensemble des individus à partir de 1517 via la Réforme initiée par Martin Luther (1483-1546). Le protestantisme développe en effet à compter de cette date l'idée de la liberté de l'âme humaine, dont il déduit que nul hormis le Christ ne peut dicter ses pensées au fidèle (LECA, 1997, p. 166). C'est sur cet aspect de son œuvre que vont rebondir les philosophes anglais.

Ce sont en effet eux qui, en prolongeant cette double évolution, vont finalement "révéler " la conception alternative de la dignité humaine. Leurs réflexions visent à donner une assise théorique aux

22 La liberté des modernes, expliquait-il, c'est « l'indépendance individuelle » par rapport au pouvoir politique, c'est-à-dire la liberté-autonomie, tandis que celle des anciens, c'est " la participation active et constante au pouvoir collectif ", c'est-à-dire la liberté-participation (CHARPENTIER, 1874, p. 261 - 268). 
aspirations de la bourgeoisie commerçante, alors florissante, à un plus grand respect de ses libertés pour mener à bien ses affaires. C'est ainsi qu'en 1690 John Locke (1632-1704) va, dans ses Deux Traités sur le gouvernement civil, recourir à la fiction du contrat social pour justifier la primauté de l'individu sur la société et l'obligation qui en découle pour cette dernière de respecter ses droits naturels, au premier rang desquels figure la liberté-autonomie ${ }^{23}$ (LOCKE, 1802). Pour lui, la fondation de la communauté n'a en effet d'autres buts que d'assurer la protection des associés. De sorte que la collectivité, et l'État qui en émane, se doivent de respecter celles de leurs libertés naturelles dont le sacrifice n'est pas indispensable à la vie commune ${ }^{24}$.

Le mot « liberté », jusque-là synonyme au plan politique de « libertéparticipation », s'enrichit ainsi d'une nouvelle signification. Or, cette dernière repose implicitement sur une nouvelle conception de la dignité humaine dont la spécificité est rétrospectivement d'investir l'homme d'un total pouvoir sur lui-même, c'est-à-dire d'en faire une source exclusive de libertés pour les individus.

De nombreuses dispositions du droit français peuvent se réclamer de cette approche. II en est notamment ainsi du droit au suicide ou, en matière de bioéthique, du droit des femmes de recourir à l'avortementet de chacun d'utiliser des moyens de contraception (préservatifs, pilule...), de refuser des soins curatifs sans bénéfice thérapeutiques, voire même de demander son euthanasie passive... Car toutes ces dispositions, aux antipodes de la conception jusnaturaliste classique, sont conformes aux implications que la conception jusnaturaliste moderne attache à la notion de dignité humaine.

Si on délaisse son point de vue, il reste toutefois possible d'expliquer l'état du droit positif par le simple attachement des individus

23 "L'homme, dans l'état de nature, est (...) libre (...), il est le seigneur absolu de sa personne » (LOCKE, 1802, VIII, § 1er).

24 « en remettant ainsi leurs privilèges naturels » les hommes, " n'ayant d'autre intention que de pouvoir mieux conserver leurs personnes, leurs libertés, leurs propriétés », "le pouvoir de la société (...) ne peut jamais (...) s'étendre plus loin que le bien public ne le demande » (LOCKE, 1802, VIII, § 10). 
à ceux de leurs droits et libertés qu'ils jugent les plus essentiels. Rien d'étonnant dès lors à ce que la conception positiviste sociologique rejoigne la conception jusnaturaliste moderne pour faire de la dignité humaine une source exclusive de droits envers autrui.

1.2 La conception positiviste sociologique ou le rejet d'une dualité de fonctions fondé sur les aspirations de la conscience collective

La conception positiviste sociologique rejette toute dualité de fonctions de la notion de dignité, dès lors que l'attachement de la masse des consciences individuelles à la défense des intérêts de chacun la conduit à en faire intrinsèquement une source de droits et de libertés envers autrui.

Forgé au début du XXe siècle par Émile Durkheim et Léon Duguit, le positivisme sociologique se différencie du jusnaturalisme par son rejet de l'idée de l'existence de droits naturels. Le seul droit dont il reconnait l'existence est le droit positif. II se rapproche en cela du positivisme juridique. Mais il s'en distingue par son refus de couper le droit de la réalité sociale dont il est issu. D'où une divergence importante : alors qu'avec le positivisme juridique toute norme doit être considérée comme du droit dès lors qu'elle a été élaborée conformément aux formes et procédures prévues à cet effet, avec le positivisme sociologique, pour être légitime, la norme doit également être conforme aux attentes de la masse des consciences individuelles (LEBRETON, 2009, p. 26; CARBONNIER, 2004, p. 27).

Avec cette nouvelle vision des choses, la dignité de la personne humaine cesse d'être une notion purement formelle pour se doubler d'une acception matérielle. Doit en effet être considéré comme portant ou non atteinte à cette dignité tout ce que l'opinion publique considère comme tel. Or, " l'attachement [du peuple français] aux Droits de l'homme et aux principes de la souveraineté nationale tels qu'ils ont été définis par la Déclaration de 1789 » et « le préambule de la Constitution de 1946 » montre que les citoyens aspirent à faire de la dignité, non une source de limitation, mais une garantie de leurs droits et libertés, 
pas seulement dans leur relation avec autrui, mais également dans celle qu'ils entretiennent avec eux-mêmes.

D'aucuns objecteront que, même en mettant de côté l'interdiction du lancé de nain, la dignité humaine fonde en droit positif un certains nombre de restrictions au pouvoir d'autodétermination que l'individu peut exercer sur lui. Et de citer la défense en principe faite à toute personne de rouler sans ceinture de sécurité ou de se droguer.

II est vrai que ces interdictions peuvent s'interpréter comme une limitation destinée à protéger son destinataire contre lui-même au nom d'une conception de la dignité qui le dépasse. Ainsi comprises, ces mesures ne seraient que l'illustration de la haute opinion que les citoyens se font de leur nature humaine. Pour séduisante qu'elle soit, cette analyse présente le défaut de reposer sur une vision trop généreuse de l'homme que démentent quotidiennement les faits. II n'y a qu'à rappeler l'existence des crimes contre l'humanité ou des génocides et leur commission quasi quotidienne pour s'en convaincre. C'est pourquoi une autre interprétation semble possible.

Plus prosaïquement, ces mesures peuvent en effet s'interpréter comme une conséquence indirecte des droits que toute personne possède envers autrui. Dès lors qu'elles portent sur des comportements qui, bien que dirigés contre soi-même, sont potentiellement de nature à porter atteinte à l'intérêt des tiers, on peut y voir le corollaire logique de la protection des droits et libertés de chacun. L'on sait en effet depuis longtemps qu'un droit revêt en réalité deux aspects. Côté pile, il garantit toute personne du concours de la puissance publique dans la défense de ses intérêts juridiquement protégés. Mais cela revient à dire que, côté face, il crée des "obligations de faire " ou " de ne pas faire " (BENTHAM apud GRZEGORCZYK et al, 1993, p. 70) à l'égard des tiers dont la violation se trouve juridiquement sanctionnée. La Déclaration de 1789 ne dit pas autre chose lorsqu'elle affirme que « l'exercice des droits (...) de chaque homme n'a de bornes que celles qui assurent aux autres membres de la société, la jouissance de ces mêmes droits » (art. 4). Or, la défense en principe faite à chacun de rouler sans ceinture de sécurité ou de se droguer représente l'envers de cette médaille. Car elle 
ne vise pas tant à protéger la personne contre elle-même qu'à protéger la dignité des tiers contre des agissements qui leur sont potentiellement nuisibles, dès lors qu'ils sont susceptibles de porter atteinte à leurs droits et libertés. L'attitude de l'automobiliste sans ceinture est potentiellement liberticide pour autrui, ne serait-ce que parce qu'il peut, en cas de choc, perdre le contrôle de son véhicule et ainsi constituer une menace pour l'intégrité physique. De même, le comportement du " junkie », l'est dans la mesure où il peut être porté à détrousser violemment le premier venu pour se procurer sa « dose » et ainsi violer son droit de propriété voire, éventuellement, son droit à l'intégrité physique ou à la vie.

C'est pourquoi la dignité humaine semble intrinsèquement source de droits : car son respect fonde le droit de chacun d'exiger des tiers un comportement qui ne mette pas en danger sa propre dignité, même lorsque ce comportement semble de prime abord dirigé contre euxmêmes.

L'approche positiviste sociologique n'est pas sans rappeler de ce point de vue l'approche jusnaturaliste moderne en ce qu'elle assigne à la dignité humaine une fonction exclusive. Mais elle s'en distingue dans la mesure où celle-ci ne consiste plus simplement à fonder le respect de la liberté-autonomie de chacun, mais l'ensemble des droits et libertés nécessaires à protéger les individus dans leurs relations mutuelles ou avec le pouvoir.

\section{Conclusion}

À la question de savoir si le principe de dignité exerce une dualité de fonctions, la théorie juridique apporte une réponse nuancée. Car tout dépend finalement de la conception du droit que l'on retient.

Si l'on admet que le droit puisse être imposé « d'en haut », par Dieu (jusnaturalisme classique) ou les autorités normatives (positivisme juridique) cette dualité semble bien réelle, car la dignité peut alors justifier tout autant de reconnaître un certain nombre de droits et libertés aux individus dans leur relation avec les tiers que de leur imposer le respect d'un certain nombre de devoirs envers eux-mêmes. 
$\mathrm{Si}$, à l'inverse, on considère que le droit vient « d'en bas », qu'il résulte d'une introspection intérieure à laquelle chacun doit procéder en raison (jusnaturalisme moderne) ou des aspirations de la conscience collective (positivisme sociologique) cette dualité ne saurait être admise. Car le concept n'a alors d'intérêt qu'autant qu'il garantit les droits ou libertés de chacun. Sans doute l'effectivité de cette garantie passe-t-elle parfois par l'interdiction de certains comportements dangereux pour soimême. Mais de telles mesures ne sont qu'une conséquence indirecte du principe; elles ne sauraient constituer sa fonction intrinsèque, qui reste de fonder les droits ou libertés des individus.

Au-delà de cette opposition, l'examen comparé de ces différentes approches est intéressant à un double titre.

Tout d'abord, en ce qu'il traduit une certaine évolution du concept de dignité qui, au-delà des soubresauts de l'histoire, reflète elle-même une certaine évolution du regard que l'homme a porté sur lui-même à travers le temps. Tandis que, sous l'Antiquité et le Moyen-âge, la notion était mise en avant pour défendre le simple droit de chacun à exister à une époque où le groupe ou le maître avait tout pouvoir sur ses membres ou féaux, elle est invoquée aux Temps modernes pour imposer le respect de la liberté individuelle à un pouvoir contraint par la religion d'autolimiter son droit de vie ou de mort sur ses sujets et, à l'époque contemporaine, pour assurer une certaine qualité de vie à tous.

L'approche positivisme sociologique permet ainsi de mettre en lumière le caractère essentiellement qualitatif de la conception contemporaine du principe de dignité : son invocation ne vise plus tant à permettre à tous de vivre (acception jusnaturaliste " classique ») ou de vivre libre (acception jusnaturaliste moderne) qu'à vivre bien. "La dignité », aujourd'hui, "c'est », pour reprendre l'expression d'un parlementaire, "le droit de ne pas vivre à genoux » (GREMETZ, M., 1999, p. 3685) $)^{25}$. zzz

\footnotetext{
25 Ainsi entendue, la promotion de l'acception contemporaine du principe de dignité traduit un approfondissement de la mission que des auteurs tels qu'Aristote $(1881, \mathrm{I}, \S 8)$ ou Locke (1802, VI, § 18) assignaient au corps politique.
} 
Cette acception permet de redonner une cohérence d'ensemble au droit positif. Tout d'abord, elle explique les dispositions ouvrant à chacun le droit de prévenir la réalisation ou de demander la réparation du préjudice, physique ou psychologique, dont il peut être victime (libertés publiques ou droits de 1 ère génération) ; et son droit à exiger certaines prestations de l'État, tel que l'accès aux soins ou à un logement décent (droits-créances ou droits de $2^{\mathrm{e}}$ génération). Ensuite, elle explique les dispositions limitant le pouvoir d'autodétermination de certains, afin de prévenir les comportements potentiellement dangereux pour les autres. Enfin, elle explique la légalité du suicide et la légalisation partielle de l'euthanasie. Car, ce droit de "bien vivre » a pour corollaire celui de mettre fin à son existence lorsque celle-ci devient une souffrance ${ }^{26}$. Sans doute certaines dispositions n'entrent-elles pas dans ce schéma, telle la condamnation par le juge des "lancés de nain ». Mais loin d'invalider l'idée de l'émergence d'une conception qualitative du principe de dignité, elles ne font que refléter l'existence d'une tension dialectique qui soustend le droit positif.

C'est en effet le second intérêt de l'examen croisé des différentes conceptions de la dignité humaine que de mettre en lumière une opposition entre les aspirations des citoyens à faire de la dignité humaine une garantie de leurs droits et libertés dans leurs relations inter personnelles et la tentation des autorités de s'en servir pour leur imposer le respect d'un certains nombres de devoirs "intra personnels ". Tout se passe comme si les dépositaires du pouvoir empruntaient via le positivisme juridique à la conception jusnaturaliste classique l'idée que des « instances supérieures » peuvent limiter la faculté d'autodétermination des individus sur eux-mêmes tandis que l'opinion publique retenait au contraire de la conception jusnaturaliste moderne l'idée qu'il revient à chacun de déterminer en raison ce que commande la notion, pour en faire collectivement une garantie efficace des droits et libertés de chacun.

26 C'est pourquoi selon nous la conception moderne du principe de dignité vise davantage à permettre à chacun de vivre bien qu' " à restituer " à tout individu " les moyens d'assurer son accomplissement personnel » (LEBRETON, 2000, p. 55) ou son "libre épanouissement » (DREYER, 2006, p. 256). 
Sans doute cette opposition complique-t-elle la compréhension du droit et pose-t-elle l'épineuse question de la légitimité de certaines normes du point de vue des conceptions jusnaturalistes et positiviste sociologique. Mais nous inviter à y réfléchir n'est pas le moindre de ses intérêts.

\section{Références}

AQUIN, Thomas d'. La somme théologique de saint Thomas d'Aquin: latin-français en regard. Paris: E. Belin, 1862. t. 8.

ARISTOTE. Éthique à Nicomaque. Paris: C. Delagrave, 1897. v. 7. . La politique. Paris: Garnier Frères, 1881.

BAS, P.; DEREPAS, L. Une atteinte intolérable à la dignité des femmes et des enfants pour la mère. Le Monde, Paris, 23 mai 2009.

BOTTINI, F. La protection des décideurs publics face au droit pénal. Paris: L. G. D. J., 2008.

CARBONNIER, J. Sociologie juridique. Paris: PUF, 2004.

CARRE DE MALBERG, R. Contribution à la théorie générale de I'État. Paris: Sirey 1920. t. 1.

CARRIERE, J.-C. La controverse de Valladolid. Paris: Plon, 1999.

CAYLA, O. Dignité humaine: le plus flou des concepts. LeMonde, Paris, p. 14-255, 31 janv. 2003.

. Jeux de nains, jeux de vilains in les droits fondamentaux de la personne humaine en 1995 et 1996. Paris: L'Harmattan 1998.

CONSTANT, Benjamin. De la liberté des Anciens comparée à celle des modernes. Paris: Charpentier, 1874. (Oeuvres politiques).

CRUVEILHIER, P. Commentaire du Code d'Hammourabi. Paris: E. Leroux, 1938. 
LE DÉRAPAGE de Brice Hortefeux à l'université d'été de l'UMP. Le Monde, Paris, 10.9.2009. Disponible: <http://www.lemonde.fr/politique/ video/2009/09/10/le-derapage-de-brice-hortefeux-a-I-universite-d-etede-I-ump_1238728_823448.html>. Accédé: 14. oct. 2012.

DREYER, E. Dignité in dictionnaire des droits fondamentaux. Paris: Dalloz, 2006.

. La dignité opposée à la personne. Paris: Dalloz, 2008.

EDELMAN, B. La dignité de la personne humaine, un concept nouveau. Paris: Dalloz, 1997.

ÉGLISE CATHOLIQUE. Concile Vatican II: Gaudium et spes, 7.12.1965. Disponible: < http://www.vatican.va/archive/hist_councils/ii_vatican_ council/documents/vat-ii_const_19651207_gaudium-et-spes_fr.html>. Accédé: 29. oct. 2012.

. Congrégation pour la doctrine de la foi. Libertatis conscientia, 22.3.1986, $\quad \mathrm{n}^{\circ}$ 27. Disponible:<http://www.vatican.va/roman_curia/ congregations/cfaith/documents/rc_con_cfaith_doc_19860322_ freedom-liberation_fr.html>. Accédé: 29. oct. 2012.

Rerum novarum. 15.5.1891. Disponible:<http://www.vatican. va/holy_father/leo_xiii/encyclicals/documents/hf_I-xiii_enc_15051891_ rerum-novarum_fr.html>. Accédé: 29. oct. 2012.

EMERI, C.; SEURIN J.-L. Chronique constitutionnelle et parlementaire française: vie et droit parlementaire. RDP, Genève, n. 3, p. 640-641, 1970.

FEDELMAN, J.-P. Faut-il protéger l'homme contre lui-même? La dignité, l'individu et la personne humaine. Droits: Revue Française de Théorie, de Philosophie et de Culture Juridiques, Hondainville, n. 48 , p. 87 $-107,2009$.

GREMETZ, M. Journal Officiel. Déb Parl. A.N. 3ème séance du 27.4.1999. p. 3658-3687. Disponible: <http://www.assemblee-nationale. fr/11/cri/html/19990220.asp>. Accédé: 14 oct. 2012.

GRZEGORCZYK, C.; MICHAUT, Troper. Le positivisme juridique, coll: la pensée juridique moderne. Paris: L. G. D. J., 1993. 
JORION, B. La dignité de la personne humaine. Revue du Droit Public et de la Science Politique en France et à l'Étranger, Paris, n. 1, p. 197-233, janv./févr. 1999.

JOUBERT, J. Pensées: maximes et essais. Paris: Le Normant, 1850.

LABORDERE, A. Les pensées de Marc Aurèle sur le Stoöcisme. Lons-le-Saunier: L. Declume, 1903.

LEBRETON Gilles. Note sous Conseil d'Etat 27 octobre 1995, Cne de Morsang-sur-Orges. Jurisprudence. Recueil Dalloz, Paris, v. 1, n.1, p. 177-180, 1996.

. Les ambigüités du droit français à l'égard de la dignité humaine. In: MÉLANGES en I'honneur de Patrice Gélard. Paris: Montchrestien, 2000. p. 1-15.

. Libertés publiques et droits de l'homme. Paris: Sirey, 2009.

LECA, A. Histoire des idées politiques. Paris: Ellipses, 1997.

LOCKE, J. Traité du gouvernement civil, VIII. Paris: C. Volland, 1802.

. Traité du gouvernement civil. Paris: C. Volland, 1802.

LUXEMBOURG. Loi du.16.3.2009. Loi relative aux soins palliatifs, à la directive anticipée et à l'accompagnement en fin de vie. Journal Officiel du Grand Duché de Luxembourg 16.3.2009, p. 610-619.

MAQUIAVEL, N. Le Prince. Paris: Garnier Frères, 1884.

MATHIEU, B. La dignité de la personne humaine: quel droit? Quel titulaire? Paris: Dalloz, 1996.

. Pour une reconnaissance de "principes matriciels" en matière de protection constitutionnelle des droits de l'homme. Paris: Dalloz, 1995.

MONTESQUIEU. De l'esprit des lois. Paris: C. Delagrave 1887.

MOUTOUH, H. La dignité de l'homme en droit. Revue du Droit Public, Paris, n. 1, p. 159-196, 1999. 
OGIEN, R. Sortir du paternalisme moralisateur. Le Monde, Paris, 23.5.2009.

RENUCCI, J.-F. Droit européen des droits de I'homme. Paris: LGDJ, 1999.

ROMAN, D. et al. Voyage au bout de la dignité. Paris: Univ. Paris I, 2004.

ROUBIER, P. Théorie générale du droit. Paris: Dalloz, 2005.

SÉRIAUX, A. Le droit naturel. Paris: PUF, 1993.

TORTURE: les mémos secrets de la CIA rendus publics. Le Monde, Paris, 16.4.2009. Disponible:<http://www.lemonde.fr/ameriques/ article/2009/04/16/les-agents-de-la-cia-ne-seront-pas-poursuivis-pourdes-actes-de-torture_1181816_3222.html>. Accédé: 29. oct. 2012.

VAUCHEZ-HENNETTE, S. Une dignitas humaine: vieilles outres, vin nouveau. Droits: Revue Française de Théorie Juridique, n. 48, p. 5985, 2009.

VEIL, S.; FRANCE. Présidence de la République. Redécouvrir le préambule de la Constitution. Paris: La Documentation Française, 2008.

VOLTAIRE. La mort de César. Paris: Garnier Frères, 1877. (Oeuvres complètes, t. 2.)

Recebido em: 03/05/12

Aprovado em: 14/05/12 